\title{
TWO EXAMPLES OF BRAUER-MANIN OBSTRUCTION TO INTEGRAL POINTS
}

\author{
ANDREW KRESCH AND YURI TSCHINKEL
}

\begin{abstract}
We give two examples of Brauer-Manin obstructions to integral points on open subsets of the projective plane.
\end{abstract}

\section{INTRODUCTION}

Let $k$ be a number field, and let $X$ be a smooth projective geometrically irreducible variety over $k$. It is well known that the existence of points of $X$ over all completions $k_{v}$ of $k$ does not imply the existence of a $k$-rational point on $X$, in general. This phenomenon is referred to as the failure of the Hasse principle. Examples of failure of the Hasse principle are known for genus 1 curves, cubic surfaces, etc. Even when the Hasse principle holds, rational points need not be dense in the set of adelic points of $X$. This phenomenon - the failure of weak approximation - is also known in many examples.

The Brauer-Manin obstruction [11, 12] often explains the failure of the Hasse principle and weak approximation. The exact sequence from class field theory

$$
0 \rightarrow \operatorname{Br}(k) \longrightarrow \bigoplus_{v} \operatorname{Br}\left(k_{v}\right) \stackrel{\sum \operatorname{inv}_{v}}{\longrightarrow} \mathbb{Q} / \mathbb{Z} \rightarrow 0
$$

(where $\sum \operatorname{inv}_{v}$ denotes the sum of local invariants) leads to the constraint

$$
X(k) \subset X\left(\mathbb{A}_{k}\right)^{\operatorname{Br}}:=\left\{\left(x_{v}\right) \in X\left(\mathbb{A}_{k}\right) \mid \sum \operatorname{inv}_{v}\left(\left.\alpha\right|_{x_{v}}\right)=0 \forall \alpha \in \operatorname{Br}(X)\right\}
$$

on the set $X\left(\mathbb{A}_{k}\right)$ of adelic points on $X$, where $\operatorname{Br}(X)$ denotes the cohomological Brauer group (see $[6])$. When $X\left(\mathbb{A}_{k}\right)^{\mathrm{Br}} \neq X\left(\mathbb{A}_{k}\right)$, then we say there is a BrauerManin obstruction to the Hasse principle in the case $X\left(\mathbb{A}_{k}\right)^{\mathrm{Br}}=\emptyset$, and to weak approximation in the case $X\left(\mathbb{A}_{k}\right)^{\mathrm{Br}} \neq \emptyset$, respectively.

For a thorough introduction to the subject, see [15]. For a survey, see [13].

The study of rational points on projective hypersurfaces is equivalent to the study of integral solutions to homogeneous Diophantine equations $f\left(x_{0}, \ldots, x_{n}\right)=0$. Many interesting Diophantine problems involve non-homogeneous equations. Their solutions can be interpreted as integral points on quasi-projective varieties.

Let $\mathfrak{o}_{k}$ be the ring of integers of $k$. Let $\mathcal{X}$ be an integral model for $X$, that is, a scheme, projective and flat over $\operatorname{Spec}\left(\mathfrak{o}_{k}\right)$ having general fiber $X$. Let $Z$ be a reduced closed subscheme of $X$, and set $U=X \backslash Z$. Then we define $\mathcal{Z}$ to be the schemetheoretic closure of $Z$ in $\mathcal{X}$ and $\operatorname{set} \mathcal{U}=\mathcal{X} \backslash \mathcal{Z}$. We then have $\mathcal{U}\left(\mathfrak{o}_{k}\right)$, the integral points of $\mathcal{U}$. By abuse of terminology, we say that a $k$-rational point of $U$ is an integral point

Date: 20 May 2008.

2000 Mathematics Subject Classification. 14G25 (primary); 14F22 (secondary).

The first author was supported by the SNF. The second author was supported by the NSF grant 0602333. 
if its (unique) extension to an $\mathfrak{o}_{k}$-point of $\mathcal{X}$ has image in $\mathcal{U}$. (This notion depends on the choice of integral model $\mathcal{X}$.) There are $S$-integral points $\mathcal{U}\left(\mathfrak{o}_{k, S}\right)$ for $S$ a finite set of non-archimedean places of $k$ (where $\mathfrak{o}_{k, S}$ denotes the ring of $S$-integral elements of $k$ ) and $v$-adic integral points $\mathcal{U}\left(\mathfrak{o}_{v}\right)$ for $v$ a non-archimedean place of $k$; the latter will be identified with $k_{v}$-points of $U$ that extend to $\mathcal{U}\left(\mathfrak{o}_{v}\right)$.

The Brauer-Manin obstruction has been extensively studied in the setting of projective varieties; see, for example, [3]. Its study in the context of open varieties is more recent and originates in the work of Colliot-Thélène and $\mathrm{Xu}$ [4], which gives a new explanation based on the Brauer-Manin obstruction for the failure of Hasse principle exhibited in [2] and [14] in the representation of integers by quadratic forms in three variables.

The insolubility of a Diophantine equation that admits solutions in $k$ and in $v$-adic integers for all non-archimedean places $v$ of $k$ can be a manifestation of the failure of the Hasse principle, or strong approximation, for a variety. (For smooth projective varieties, strong and weak approximation are the same.) This paper provides examples of this, in which there is an explanation via the Brauer-Manin obstruction:

$$
\mathcal{U}\left(\mathfrak{o}_{k}\right) \subset\left(\prod_{v \nmid \infty} \mathcal{U}\left(\mathfrak{o}_{v}\right) \times \prod_{v \mid \infty} U\left(k_{v}\right)\right)^{\operatorname{Br}(U)},
$$

where the set on the right is the set of tuples of adelic points, integral at all nonarchimedean places, whose sum of local invariants is zero with respect to every element of $\operatorname{Br}(U)$. The formulation of the obstruction, and in particular the use of $\operatorname{Br}(U)$ rather than $\operatorname{Br}(\mathcal{U})$, follows Colliot-Thélène and $\mathrm{Xu}$; see $[4, \S 1]$.

This note is inspired by lectures of Colliot-Thélène on his joint work with Xu. As in their work, we take $U$ to be the complement of a geometrically irreducible smooth divisor $D$ on a surface $X$. In their work, $X$ is a quadric surface and $D$ a hyperplane section. Here for simplicity we take $k=\mathbb{Q}$ and $X=\mathbb{P}^{2}$, so that if a homogeneous $f \in \mathbb{Z}[x, y, z]$ defines $D$ (and $\mathcal{D})$, then elements of $\mathcal{U}(\mathbb{Z})$ correspond to triples of integers $(x: y: z)$ (up to a factor \pm 1 ) such that $f(x, y, z)= \pm 1$, where $\mathcal{U}=\mathbb{P}_{\mathbb{Z}}^{2} \backslash \mathcal{D}$. The geometric Brauer group of $U$ is understood by an exact sequence [1] that reduces in this case to an isomorphism, the ramification map:

$$
\operatorname{Br}(U \otimes \overline{\mathbb{Q}}) \stackrel{\sim}{\rightarrow} H^{1}(D \otimes \overline{\mathbb{Q}}, \mathbb{Q} / \mathbb{Z}) .
$$

Our $D \subset \mathbb{P}^{2}$ will admit unramified coverings over $\mathbb{Q}$ such that known constructions of algebras representing ramified Brauer group elements can be carried out over $\mathbb{Q}$. For the local analysis we must restrict the Brauer group elements to points of $U(\mathbb{R})$ and to the $p$-adic integral points in $U\left(\mathbb{Q}_{p}\right)$. These correspond to triples $(x: y: z)$ of reals satisfying $f(x, y, z) \in \mathbb{R}^{*}$, and $p$-adic integers satisfying $f(x, y, z) \in \mathbb{Z}_{p}^{*}$, respectively.

\section{Cubic}

This section is devoted to the following example, concerning rational and integral points on the complement of a plane cubic curve over $\mathbb{Q}$.

Example 1. For the Diophantine equation

$$
y^{2} z-(4 x-z)\left(16 x^{2}+20 x z+7 z^{2}\right)=1
$$

we have:

(i) there are solutions in $p$-adic integers for all primes $p$;

(ii) there are solutions in $\mathbb{Q}$; 
(iii) there are no solutions in $\mathbb{Z}$.

There is the rational solution $(1 / 4,1,1)$, which is a $p$-adic integral solution for $p \neq 2$. A 2-adic integral solution is $(0,0, \sqrt[3]{1 / 7})$, so statements (i) and (ii) are established.

The proof of (iii) is more subtle and uses the Brauer group. Letting the polynomial

$$
f=y^{2} z-(4 x-z)\left(16 x^{2}+20 x z+7 z^{2}\right)
$$

define the divisor $D \subset \mathbb{P}^{2}$ (and $\mathcal{D} \subset \mathbb{P}_{\mathbb{Z}}^{2}$ ) and setting $U=\mathbb{P}^{2} \backslash D$ (and $\mathcal{U}=\mathbb{P}_{\mathbb{Z}}^{2} \backslash \mathcal{D}$ ) we will exhibit a 2-torsion Brauer group element $A \in \operatorname{Br}(U)$ whose pull-back to $\operatorname{Br}(U \otimes \overline{\mathbb{Q}})$ has prescribed (nontrivial) image under the ramification map. With $A$, we are able to deduce from (1) a congruence condition on integral points of $\mathcal{U}$ that is incompatible with (2).

Remark 1. Since $f$ is homogeneous of odd degree, the insolubility of (2) in integers implies $\mathcal{U}(\mathbb{Z})=\emptyset$. Therefore, from statements (i)-(iii) of Example 1 we obtain a Brauer-Manin obstruction to the Hasse principle over $\mathbb{Z}$.

Remark 2. The fundamental group of $U \otimes \overline{\mathbb{Q}}$ is cyclic of order 3 by [16, Theorem 8$]$ (which treats the topological fundamental group) and [7, (XII.5.2), (XIII.4.6)] (which provides the link to the algebraic fundamental group). Hence a universal cover of $U \otimes \overline{\mathbb{Q}}$ is $\widetilde{U} \otimes \overline{\mathbb{Q}}$, where $\widetilde{U}$ is the affine open subscheme defined by $t \neq 0$ of the cubic surface $\widetilde{X}$ with defining equation $f(x, y, z)=t^{3}$, and the covering map is given by $(x: y: z: t) \mapsto(x: y: z)$. For the degree 3 extension of function fields $\overline{\mathbb{Q}}(U) \rightarrow \overline{\mathbb{Q}}(\widetilde{U})$, there are restriction and corestriction maps on Brauer groups, and restriction followed by corestriction acts as multiplication by 3 on $\operatorname{Br}(\overline{\mathbb{Q}}(U))$. Nontriviality of the pullback of $A$ to $\operatorname{Br}(U \otimes \overline{\mathbb{Q}}$ ) (see Lemma 2.1, below) implies: the 2-torsion element $A$ remains nontrivial, and hence ramified $(\operatorname{Br}(\widetilde{X} \otimes \overline{\mathbb{Q}})=0$ since $\widetilde{X}$, a cubic surface, is geometrically rational), upon pull-back to the geometric universal cover.

Remark 3. We can state an integer-point analogue of a basic obstruction to rational points coming from torsor theory and check that it does not obstruct integral points on $\mathcal{U}$. Letting the group $\mu_{3}$ of cube roots of unity act on the variety $\widetilde{U}$ of Remark 2 by multiplication on the $t$ coordinate gives $\widetilde{U}$ the structure of $U$-torsor under $\mu_{3}$. Torsor theory, described in [15, §2.2], supplies arithmetic twists $\pi_{\gamma}: \widetilde{U}_{\gamma} \rightarrow U$ indexed by $\gamma \in H^{1}\left(\mathbb{Q}, \mu_{3}\right)$, with the property that $U(\mathbb{Q})$ is the disjoint union of the images in $U$ of the $\widetilde{U}_{\gamma}(\mathbb{Q})$. Obstructions based on the consequence that $U(\mathbb{Q})=\emptyset$ if $\widetilde{U}_{\gamma}(\mathbb{Q})=\emptyset$ for all $\gamma$ are called descent obstructions; cf. [15, §5.3]. An easy integer-point descent obstruction states: if, for every $\gamma \in H^{1}\left(\mathbb{Q}, \mu_{3}\right)$, there exists a prime $p_{\gamma}$ such that $\pi_{\gamma}\left(\widetilde{U}_{\gamma}\left(\mathbb{Q}_{p_{\gamma}}\right)\right)$ contains no $p_{\gamma}$-adic integral points, then $\mathcal{U}(\mathbb{Z})=\emptyset$. It follows from the proof of $[15$, Proposition 5.3.2] that there are only finitely many classes $\gamma$ such that $\pi_{\gamma}\left(\widetilde{U}_{\gamma}\left(\mathbb{Q}_{p}\right)\right)$ contains a $p$-adic integral point for every prime $p$ (the properness hypothesis of the statement is used only for the lifting of rational points to integral points). For the untwisted $\widetilde{U}_{0}=\widetilde{U}$, there exist $p$-adic integral points in $\pi_{0}\left(\widetilde{U}_{0}\left(\mathbb{Q}_{p}\right)\right)$ for every prime $p$ by statement (i).

The curve $D$ is an elliptic curve over $\mathbb{Q}$ with group structure $D(\mathbb{Q}) \cong \mathbb{Z} / 2 \mathbb{Z}$, as is readily computed. Therefore, there is a unique (up to isomorphism) nontrivial unramified cover $\widetilde{D} \otimes \overline{\mathbb{Q}} \rightarrow D \otimes \overline{\mathbb{Q}}$ that arises by base change from some cover $\widetilde{D} \rightarrow D$ defined over $\mathbb{Q}$ of degree 2 . 
Lemma 2.1. The class in $\operatorname{Br}(\mathbb{Q}(U))$ of the quaternion algebra

$$
\left(y^{2} z^{-2}-(4 x-z)\left(16 x^{2}+20 x z+7 z^{2}\right) z^{-3},(4 x-z) z^{-1}\right)
$$

is the restriction of a Brauer group element $A \in \operatorname{Br}(U)$, such that the pull-back of $A$ to $\operatorname{Br}(U \otimes \overline{\mathbb{Q}})$ is sent by the ramification map to the class of the extension $\overline{\mathbb{Q}}(D) \rightarrow \overline{\mathbb{Q}}(\widetilde{D})$.

Proof. The assertion about $\operatorname{Br}(U)$ uses the fact, immediate from [6, (III.6.2)], that the image of $\operatorname{Br}(U)$ in $\operatorname{Br}(\mathbb{Q}(U))$ is the kernel of a ramification map $\operatorname{Br}(\mathbb{Q}(U)) \rightarrow$ $\bigoplus_{u \in U^{(1)}} H^{1}\left(\mathbf{k}_{u}, \mathbb{Q} / \mathbb{Z}\right)$, where the sum is over generic points $u$ of codimension 1 subvarieties of $U$, with $\mathbf{k}_{u}$ the residue field at such a point $u$. The argument of $[1, \S 3]$ supplies a concrete description of this map (since the base field $\mathbb{Q}$ is not algebraically closed, the right-hand 0 in the exact sequence [1, (3.2)] needs to be replaced by $\left.H^{3}\left(\mathbb{Q}(U), \mathbb{G}_{m}\right)\right)$ : for $f, g \in \mathbb{Q}(U)^{*}$, if $f, g \in \mathcal{O}_{U, u}^{*}$ then $(f, g) \in \operatorname{Br}(U)$ is in the kernel of the ramification map at $u$, while if $f \in \mathcal{O}_{U, u}^{*}$ and $g$ is a uniformising element of the discrete valuation ring $\mathcal{O}_{U, u}$, then $(f, g)$ is in the kernel of the ramification map when the residue class $\bar{f}$ of $f$ in $\mathbf{k}_{u}$ is a square and otherwise maps to the class of $\mathbf{k}_{u} \rightarrow \mathbf{k}_{u}\left(\bar{f}^{1 / 2}\right)$. Therefore, $A$ is in the kernel of the ramification map at the line $4 x-z=0$. Rewriting $(3)$ in $\operatorname{Br}(\mathbb{Q}(U))$ as

$$
\begin{aligned}
\left(-z y^{-1}+(4 x-z)\left(16 x^{2}+20 x z+7 z^{2}\right) y^{-3}, z y^{-1}\right) \\
+\left(z^{2} y^{-2}-(4 x-z)\left(16 x^{2}+20 x z+7 z^{2}\right) z y^{-4},(4 x-z) y^{-1}\right),
\end{aligned}
$$

we see that $A$ is in the kernel of the ramification map at $z=0$. At other $u \in U^{(1)}$, the rational functions in $(3)$ are both units in $\mathcal{O}_{U, u}$.

The algebra $(3)$, over $\overline{\mathbb{Q}}(U)$, is the explicitly given symbol algebra in $[9$, proof of the Theorem]. There the image under the ramification map is computed and found to be as claimed.

The local analysis of the class $A$ is simplified by the following observation.

Lemma 2.2. The element $A \in \operatorname{Br}(U)$ from Lemma 2.1 can be extended to $\operatorname{Br}(\mathcal{U} \otimes$ $\mathbb{Z}[1 / 2])$.

Proof. Lemma 2.1 implies that for suitable $N$ there exists on $\mathcal{U}_{1}=\mathcal{U} \otimes \mathbb{Z}[1 / 2 N]$ an $\mathcal{A}_{1} \in \operatorname{Br}\left(\mathcal{U}_{1}\right)$ extending $A$. If we define $\mathcal{U}_{2}$ to be the complement in $\mathcal{U} \otimes \mathbb{Z}[1 / 2]$ of the union of $\mathcal{D}$ and the scheme defined by $(4 x-z) z=0$, then (3) defines $\mathcal{A}_{2} \in \operatorname{Br}\left(\mathcal{U}_{2}\right)$ also extending $A$. The restrictions of $\mathcal{A}_{1}$ and $\mathcal{A}_{2}$ to $\operatorname{Br}\left(\mathcal{U}_{1} \cap \mathcal{U}_{2}\right)$ are equal since $\mathcal{U} \otimes \mathbb{Z}[1 / 2]$ is regular and $\mathcal{A}_{1}$ and $\mathcal{A}_{2}$ extend the same element of $\operatorname{Br}(\mathbb{Q}(U))$. Therefore, by the Mayer-Vietoris sequence, $A$ extends to an element of $\operatorname{Br}\left(\mathcal{U}_{1} \cup \mathcal{U}_{2}\right)$. By a purity result of Gabber for three-dimensional regular schemes [5, Theorem $\left.2^{\prime}\right]$, the restriction map $\operatorname{Br}(\mathcal{U} \otimes \mathbb{Z}[1 / 2]) \rightarrow \operatorname{Br}\left(\mathcal{U}_{1} \cup \mathcal{U}_{2}\right)$ is an isomorphism.

At any 2-adic integral point $(x: y: z)$ of $\mathcal{U}$ satisfying

$$
y \equiv 0 \quad(\bmod 2) \quad \text { and } \quad z \equiv 1 \quad(\bmod 2),
$$

direct evaluation reveals that $A$ is nonzero at $(x: y: z) \in U\left(\mathbb{Q}_{2}\right)$. By Lemma 2.2, $A$ vanishes at all $p$-adic integral points of $\mathcal{U}$, for any odd prime $p$. Since $U(\mathbb{R})$ is connected, the behaviour of $A$ at real points of $U$ is revealed by evaluation at a single point, and we find that $A$ vanishes at real points. Therefore, by condition (1), $\mathcal{U}$ has no integral points satisfying (4). Any integral solution to (2) would have to satisfy (4), as we see by reduction modulo 2. Therefore, (2) has no integral solutions, and statement (iii) is established. 


\section{QuARTiC}

In this section, we study the complement of a quartic curve in the projective plane over $\mathbb{Q}$.

Example 2. For the Diophantine equation

$$
-2 x^{4}-y^{4}+18 z^{4}=1
$$

we have:

(i) there are solutions in p-adic integers for all primes $p$;

(ii) there are solutions in $\mathbb{Q}$;

(iii) there are no solutions in $\mathbb{Z}$.

The rational solution $(1 / 2,0,1 / 2)$ is a $p$-adic integral solution for $p \neq 2$, and a 2 -adic integral solution is $(0, \sqrt[4]{17}, 1)$. Thus, (i) and (ii) are established.

Let $D \subset \mathbb{P}^{2}$ and $\mathcal{D} \subset \mathbb{P}_{\mathbb{Z}}^{2}$ be defined by the polynomial

$$
f=-2 x^{4}-y^{4}+18 z^{4},
$$

and set $U=\mathbb{P}^{2} \backslash D$ and $\mathcal{U}=\mathbb{P}_{\mathbb{Z}}^{2} \backslash \mathcal{D}$. For (iii), we will use a 2-torsion class $A \in \operatorname{Br}(U)$.

The equation for $D$ can be transformed to $\left(4 x^{2}-y^{2}\right)^{2}+2\left(x^{2}+2 y^{2}+9 z^{2}\right)\left(x^{2}+\right.$ $\left.2 y^{2}-9 z^{2}\right)=0$, so $\mathbb{Q}(D) \rightarrow \mathbb{Q}(D)\left(\sqrt{x^{2}+2 y^{2}+9 z^{2}} / x\right)$ describes an unramified degree 2 cover $\widetilde{D} \rightarrow D$.

Lemma 3.1. The class in $\operatorname{Br}(\mathbb{Q}(U))$ of the quaternion algebra ${ }^{1}$

$$
(f h,-g h),
$$

with

$$
g=-28 x^{2}-36 x y+7 y^{2}+72 z^{2} \quad \text { and } \quad h=-25 x^{2}+16 x y-22 y^{2}+81 z^{2},
$$

is the restriction of an element $A \in \operatorname{Br}(U)$. The pull-back of $A$ to $\operatorname{Br}(U \otimes \overline{\mathbb{Q}})$ is sent by the ramification map to the class of the extension $\overline{\mathbb{Q}}(D) \rightarrow \overline{\mathbb{Q}}(\widetilde{D})$.

Proof. For the first claim it suffices by [6, (III.6.2)] to show that $A$ is in the kernel of the ramification map at the divisors on $U$ defined by $g$ and by $h$. Since $(f h,-g h)=$ $(f, g)+(f,-h)+(g, h)$ in $\operatorname{Br}(\mathbb{Q}(U))$, this reduces to the assertions that $f g$ is a square modulo $h$ and $f h$ is a square modulo $g$, and these assertions can be verified directly.

The algebra $(6)$, over $\overline{\mathbb{Q}}(U)$, is that given in [10, proof of Proposition 1.3(iii)], applied to the ramification locus $D \otimes \overline{\mathbb{Q}}$ (after linear change of coordinates that eliminates the $y^{4}$ term from $\left.f\right)$. The image of the pull-back of $A$ to $\operatorname{Br}(U \otimes \overline{\mathbb{Q}})$ under the ramification map is calculated and found to be as claimed.

Lemma 3.2. The element $A \in \operatorname{Br}(U)$ from Lemma 3.1 can be extended to $\operatorname{Br}(\mathcal{U} \otimes$ $\mathbb{Z}[1 / 2])$.

Proof. The element $A$ extends over $\mathcal{U} \otimes \mathbb{Z}[1 / N]$ for some integer $N$, as well as over the open subscheme of $\mathcal{U} \otimes \mathbb{Z}[1 / 2]$ where $f, g$, and $h$ are nonvanishing, and hence over their union by the Mayer-Vietoris sequence. Gabber's purity result $\left[5\right.$, Theorem $\left.2^{\prime}\right]$ completes the proof.

\footnotetext{
${ }^{1}$ We take the liberty here of writing homogeneous functions of even degree rather than rational functions.
} 
By direct evaluation, we see that for $(x: y: z) \in \mathcal{U}\left(\mathbb{Z}_{2}\right)$ satisfying

$$
x \equiv 0 \quad(\bmod 2), \quad y \equiv 1 \quad(\bmod 2), \quad \text { and } \quad z \equiv 1 \quad(\bmod 2),
$$

$A$ is nonzero at $(x: y: z) \in U\left(\mathbb{Q}_{2}\right)$. By Lemma $3.2, A$ vanishes at $p$-adic integral points of $\mathcal{U}$ for $p$ odd. Evaluation at one point in each of the two connected components of $U(\mathbb{R})$ reveals that $A$ vanishes at all real points of $U$. Therefore, the constraint (1) dictates that $\mathcal{U}$ has no integral points satisfying (7). Any integral solution to (5) would have to satisfy (7), so (iii) is established.

Remark 4. Since $(0: 1: 0) \in \mathcal{U}(\mathbb{Z})$, Example 2 furnishes a Brauer-Manin obstruction to strong approximation of integral points, as formulated in $[8, \S 2]$.

Remark 5. There is a tower of projective varieties and open subvarieties

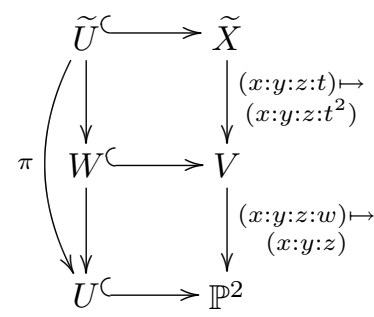

where $\tilde{X}$ is defined by $f(x, y, z)=t^{4}$ in $\mathbb{P}^{3}, V$ is defined by $f(x, y, z)=w^{2}$ in weighted projective space $\mathbb{P}(1,1,1,2)$, and $\widetilde{U} \subset \widetilde{X}$ and $W \subset V$ are defined by $t \neq 0$ and $w \neq 0$, respectively. By [16, Theorem 8] (which treats the topological fundamental group) and $[7,(X I I .5 .2)$, (XIII.4.6)] (which provides the link to the algebraic fundamental group), $\widetilde{U} \otimes \overline{\mathbb{Q}}$ is a geometric universal cover of $U$. The pull-back of $A$ to $\operatorname{Br}(W \otimes \overline{\mathbb{Q}})$ is seen by direct evaluation to lie in the kernel of the ramification map for $W \otimes \overline{\mathbb{Q}}$, and hence the pull-back of $A$ to the geometric universal cover is unramified and, in fact, trivial (since $\operatorname{Br}(V \otimes \overline{\mathbb{Q}})=0$ ).

Remark 6. The obstruction mentioned in Remark 4 is not, to the authors' knowledge, a consequence of any descent obstruction coming from $U$-torsors under finite algebraic groups. Remark 5 exhibits a $U$-torsor $\widetilde{U}$ under the group $\mu_{4}$ of fourth roots of unity, acting by multiplication on the $t$ coordinate. For every prime $p$, the set $\pi\left(\widetilde{U}\left(\mathbb{Q}_{p}\right)\right)$ contains $p$-adic integral points by statement (i), and some of these indeed satisfy $(7)$ when $p=2$.

Acknowledgement. The authors are grateful to the referee for suggestions that led to significant improvements in the presentation of this material.

\section{REFERENCES}

[1] M. Artin and D. Mumford, 'Some elementary examples of unirational varieties which are not rational', Proc. London Math. Soc. (3) 25 (1972) 75-95.

[2] M. Borovoi and Z. Rudnick, 'Hardy-Littlewood varieties and semisimple groups', Invent. Math. 119 (1995) 37-66.

[3] J.-L. Colliot-Thélène, D. Kanevsky, and J.-J. Sansuc, 'Arithmétique des surfaces cubiques diagonales', Diophantine approximation and transcendence theory, Lecture Notes in Mathematics 1290 (ed. G. Wüstholz; Springer, Berlin, 1987) 1-108.

[4] J.-L. Colliot-Thélène and F. Xu, 'Brauer-Manin obstruction for integral points of homogeneous spaces and representation by integral quadratic forms', Compositio Math., to appear. 
[5] O. Gabber, 'Some theorems on Azumaya algebras', Groupe de Brauer, Lecture Notes in Mathematics 844 (ed. M. Kervaire and M. Ojanguren; Springer, Berlin, 1981) 129-209.

[6] A. Grothendieck, 'Le groupe de Brauer, I-III', Dix exposés sur la cohomologie des schémas, Advanced Studies in Pure Mathematics 3 (North-Holland, Amsterdam, 1968) 46-188.

[7] A. Grothendieck, Revêtements étales et groupe fondamental (SGA 1), Documents Mathématiques 3, (Société Mathématique de France, Paris, 2003).

[8] B. Hassett and Yu. Tschinkel, 'Approximation at places of bad reduction for rationally connected varieties', Pure Appl. Math. Q. 4 (2008) 743-766.

[9] B. Jacob, 'Division algebras which only ramify along a hyperelliptic curve in $\mathbb{P}^{2}$, J. Algebra 242 (2001) 720-728

[10] B. È. Kunyavskiŭ, L. H. Rowen, S. V. Tikhonov, and V. I. Yanchevskiŭ, 'Division algebras that ramify only on a plane quartic curve', Proc. Amer. Math. Soc. 134 (2006) 921-929.

[11] Yu. I. Manin, 'Le groupe de Brauer-Grothendieck en géométrie diophantienne', Actes du congrès international des mathématiciens, Nice, 1970, vol. 1 (Gauthier-Villars, Paris, 1971) 401-411.

[12] Yu. I. Manin, Cubic forms: algebra, geometry, arithmetic (North-Holland, Amsterdam, 1974).

[13] E. Peyre, 'Obstructions au principe de Hasse et à l'approximation faible', Séminaire Bourbaki, 2003-2004, Astérisque 299 (Société Mathématique de France, Paris, 2005) Exp. No. 931, 165193.

[14] R. Schulze-Pillot and F. Xu, 'Representations by spinor genera of ternary quadratic forms', $A l$ gebraic and arithmetic theory of quadratic forms, Contemporary Mathematics 344 (ed. R. Baeza et al.; American Mathematical Society, Providence, RI, 2004) 323-337.

[15] A. N. Skorobogatov, Torsors and rational points (Cambridge University Press, Cambridge, 2001).

[16] O. Zariski, 'On the problem of existence of algebraic functions of two variables possessing a given branch curve', Amer. J. Math. 51 (1929) 305-328.

Institut für Mathematik, Universität Zürich, Winterthurerstrasse 190, CH-8057 Zürich, SWITZERLAND

E-mail address: andrew.kresch@math.unizh.ch

Courant Institute, 251 Mercer Street, New York, NY 10012, USA

E-mail address: tschinkel@cims.nyu.edu 